\title{
LISzyme Biosensors: DNAzymes Embedded in an Anti-Biofouling Platform for Hands-Free Real-Time Detection of Bacterial Contamination in Milk
}

Hanie Yousefi ${ }^{\dagger}{ }^{\dagger, 2}$, Sahar Esmaeili Samani ${ }^{\dagger, 1}$, Shadman Khan ${ }^{3}$, Akansha Prasad ${ }^{1,3}$, Amid Shakeri $^{4}$, Yingfu Li ${ }^{4,5}$, Carlos D. M. Filipe ${ }^{1, *}$, Tohid F. Didar ${ }^{3,5,6, *}$.

${ }^{1}$ Department of Chemical Engineering, ${ }^{3}$ School of Biomedical Engineering, ${ }^{4}$ Department of Mechanical Engineering, ${ }^{5}$ Department of Biochemistry and Biomedical Sciences, ${ }^{6}$ Institute for Infectious Disease Research (IIDR), McMaster University, Hamilton, Ontario L8S 4L8, Canada

${ }^{2}$ Leslie Dan Faculty of Pharmacy, University of Toronto, Toronto, Ontario M5S 3M2, Canada

\section{Corresponding Authors}

Professor Carlos D. M. Filipe (filipec@mcmaster.ca) and Professor Tohid F. Didar (didar@mcmaster.ca)

\section{Table of Contents:}

Fluorescence image of the FAM-labeled single-stranded DNA and milk.

TAMRA-labeled DNAzyme cleavage activity in presence of $E$. coli cells

The sequences of and modifications to all oligonucleotides used in this work

Calculating the density of immobilized DNAzymes on the LISzyme sensor 


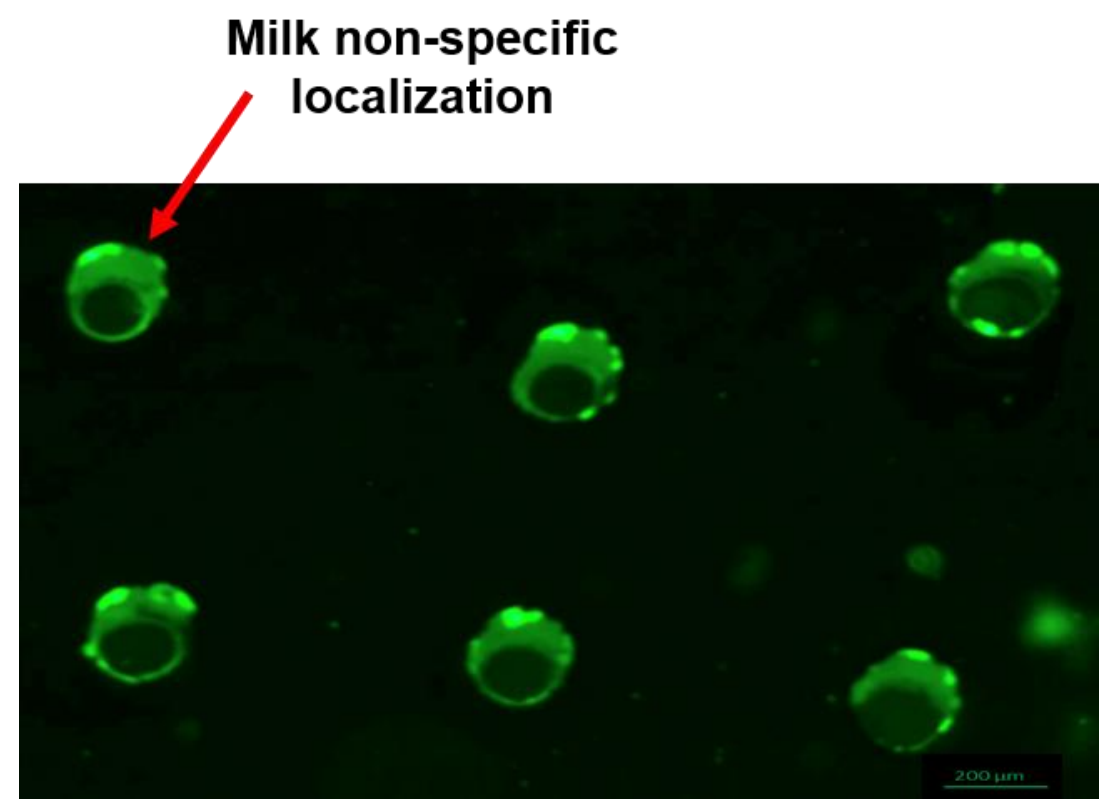

Figure S1. Fluorescence image of the FAM-labeled single-stranded DNA and milk. Image of DNA in milk under green fluorescence illumination which shows milk nonspecific fouling around DNA printed regions and its green fluorescence. 


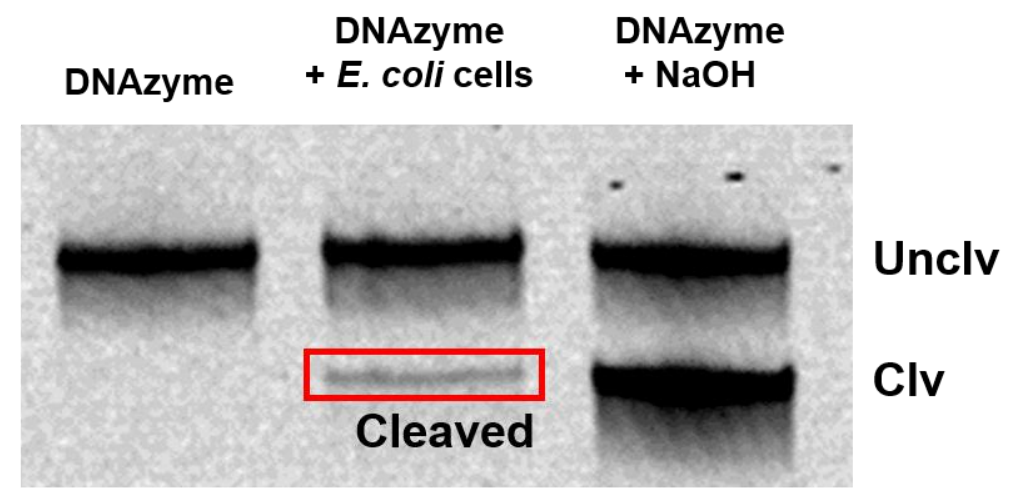

Figure S2. TAMRA-labeled DNAzyme cleavage activity in presence of CIM E. coli cells. Clv (cleaved) and Unclv (uncleaved) represent the cleavage product and the full-length DNAzyme respectively. Cleaved band in presence of E. coli cells is indicated with a red box. Final concentration of $2.5 \times 10^{3} \mathrm{CFU} / \mathrm{mL}$ of bacteria is used to demonstrate cleavage of DNAzyme with low traces of the target protein. We have observed $8 \%$ cleavage for the probes which is an acceptable ratio compared to the current literature and compared to gold-standard FAM labeled DNAzyme. ${ }^{1,2}$ It shows the modifications applied to the probe did not affect its activity. 
Table S1. The sequences of and modifications to all oligonucleotides used in this work.

\begin{tabular}{|c|c|c|}
\hline Name & Sequence & Note \\
\hline NH-EC1 & $\begin{array}{l}5^{\prime}- \\
\mathrm{NH}_{2} \text { TTTTTCACGGATCCTGACAAGGATGTGGTTGTCGAGAC } \\
\text { CTGCGACCGGAACACTACACTGTGTGGGATGGATTTCTTTAC } \\
\text { A } \\
\text { GTTGTGTGCAGCTCCGTCCG-3' }\end{array}$ & $\begin{array}{l}\text { Amine- } \\
\text { labeled } \\
\text { DNAzyme } \\
\text { sequence } \\
\text { for } E \text {. coli } \\
\quad \mathrm{K} 12\end{array}$ \\
\hline TS1 & $\begin{array}{l}5^{\prime} \text {-ACTCTTCCTAGCTrAQGGTTCGATCAAGA-3' } \\
\text { (T: TAMRA-dT; rA: riboadenosine; Q: BHQ2-dT) }\end{array}$ & $\begin{array}{l}\text { Fluorogeni } \\
\text { c substrate }\end{array}$ \\
\hline LT & $5^{\prime}$-CTAGGAAGAGTCGGACGGAGCTG-3' & $\begin{array}{l}\text { Ligation } \\
\text { template } \\
\text { for ligating } \\
\text { NH-EC1 to } \\
\text { TS1 }\end{array}$ \\
\hline RFD-EC1 & $\begin{array}{l}5^{\prime} \text {-NH }{ }_{2} \text { TTTTTCACGGATCCTGACAAGGATGTGGTTGTCGAG } \\
\text { ACCTGCGACCGGAACACTACACTGTGTGGGATGGATTTCTTT } \\
\text { ACAGTTGTGTGCAGCTCCGTCCGACTCTTCCTAGCTrAQGGT } \\
\text { TCGATCAAGA-3' }\end{array}$ & $\begin{array}{l}\text { Complete } \\
\text { DNAzyme } \\
\text { sequence } \\
\text { including } \\
\text { NH-EC1 } \\
\text { and TS1 }\end{array}$ \\
\hline TRDNA & 5'-/5AmMC12/TTT TTC ACG GAT CCT GAC AAG GAT/3TEX615/ & $\begin{array}{l}\text { Texas red- } \\
\text { labeled } \\
\text { ssDNA } \\
\text { sequence }\end{array}$ \\
\hline $\begin{array}{c}\text { FAMDN } \\
\text { A }\end{array}$ & 5'-15AmMC12/TTT TTC ACG GAT CCT GAC AAG GAT/3TFAM/ & $\begin{array}{l}\text { FAM- } \\
\text { labeled } \\
\text { ssDNA } \\
\text { sequence }\end{array}$ \\
\hline
\end{tabular}




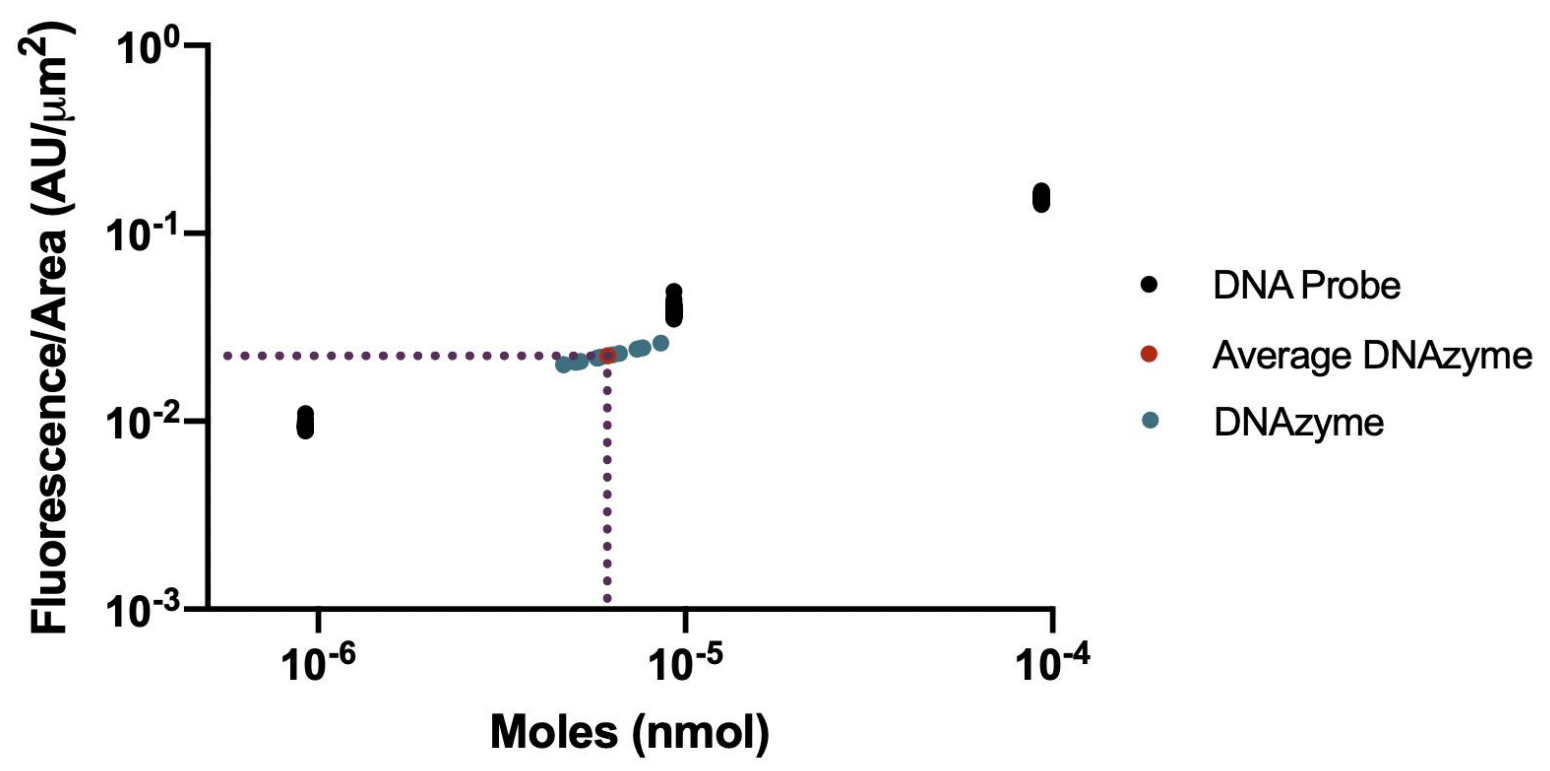

Figure S3 Calculating the density of immobilized DNAzymes on the LISzyme sensor. Fluorescently labelled DNA probes (TRDNA) were printed on fluorosilane -treated slides at known concentrations and volumes. They were then imaged immediately without washing. Fluorescence was measured over a standard area of 20,106 $\mu \mathrm{m}^{2}$. Dividing the fluorescence intensity by the standard area provided the fluorescence per unit area. By graphing the moles of DNA probe against fluorescence per unit area, a calibration curve between the two parameters was created. Linear regression yielded an equation for the relationship. DNAzymes were then printed, washed, and cleaved using $\mathrm{NaOH}$ to achieve maximal fluorescence signal. Fluorescence was measured and divided by the standard unit area. The aforementioned equation was used to calculate the moles of DNAzyme present on the surfaces - approximately $8 \times 10^{-6}$ nanomoles per microarray spot. 
a)

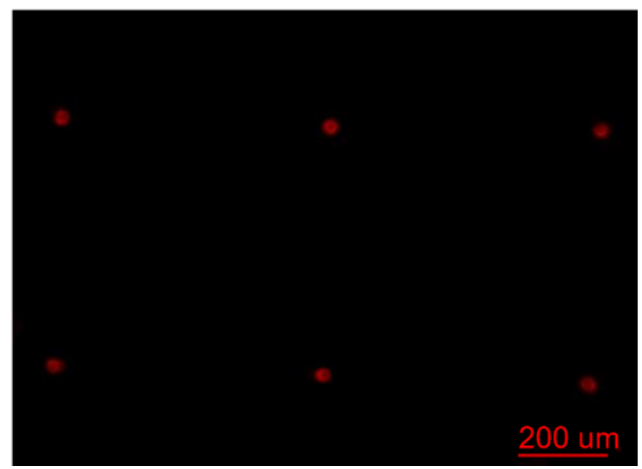

b)

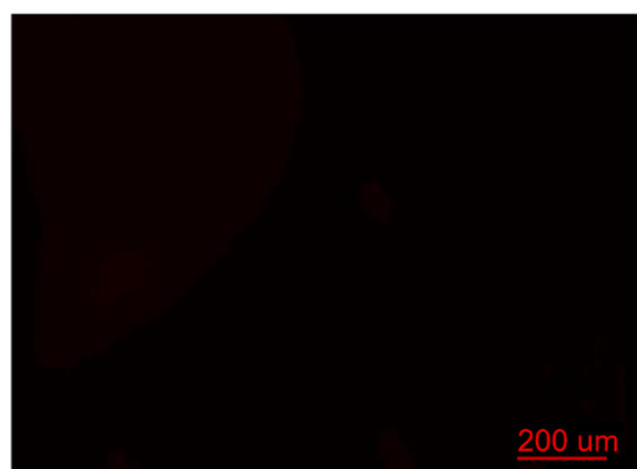

Figure S4. Importance of epoxy for DNAzyme printing onto the surfaces. Fluorescence image of the surface with TAMRA-labeled DNAzyme without epoxy before washing (a), and after washing (b). Absence of fluorescence after washing shows that DNAzyme cannot bind to the surface without epoxy.

Table S2. The long-term stability of the sensor arrays in milk. The surfaces are studied for the stability/change in their contact angle while being kept in milk for a week ay $4^{\circ} \mathrm{C}$ and compared to freshly made surfaces. The results showed that the lubricant layer is stable in milk, after one week of incubation.

\begin{tabular}{|c|c|c|c|c|}
\hline Day & \multicolumn{2}{|l|}{ Condition } & Average Contact Angle & Sliding Angle \\
\hline \multirow{2}{*}{1} & \multirow{4}{*}{ No submersion } & No lub & 116.83 & $>5$ \\
\hline & & Lub & 106.83 & $<5$ \\
\hline \multirow{2}{*}{7} & & No lub & 113 & $>5$ \\
\hline & & Lub & 104.16 & $<5$ \\
\hline 1 & \multirow{2}{*}{1 week submersion in milk } & Lub & 109.66 & $<5$ \\
\hline 7 & & Lub & 100.33 & $<5$ \\
\hline
\end{tabular}

$\mathbf{N}=6$ for each condition 


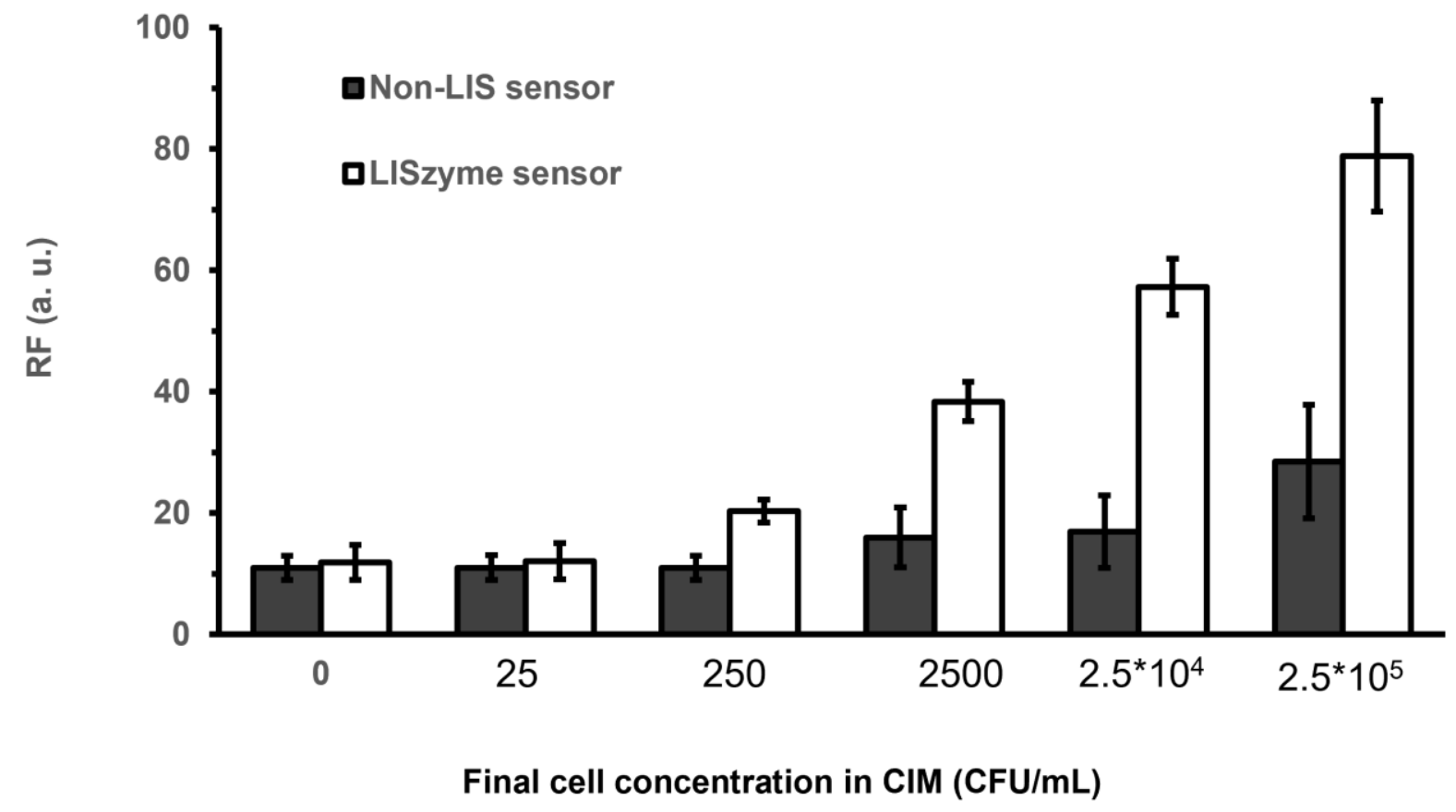

Figure S5. Comparison of the signal change of the LISzyme and non-LIS sensors in milk spiked with CIM from E. coli. The concentrations refer to the final equivalent concentration of E. coli cells in the final sample containing the mixture of milk, buffer and CIM. The surfaces were incubated with each of these mixtures for 1 hour at room temperature. 


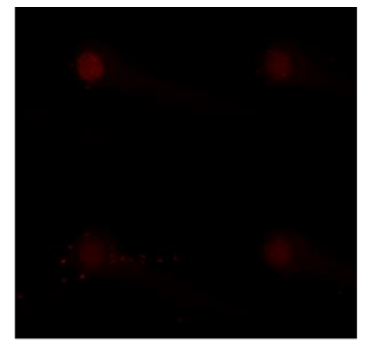

Day 1 - untreated sensors

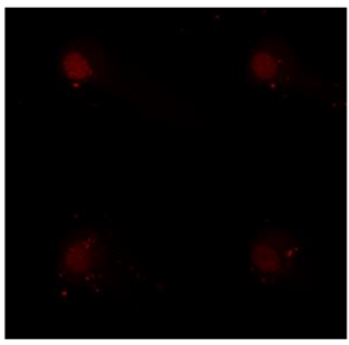

Day 7 - untreated sensors

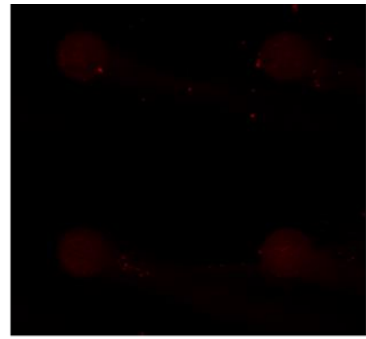

Day 1 - negative control

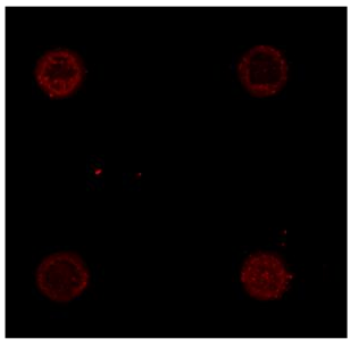

Day 7 - negative control

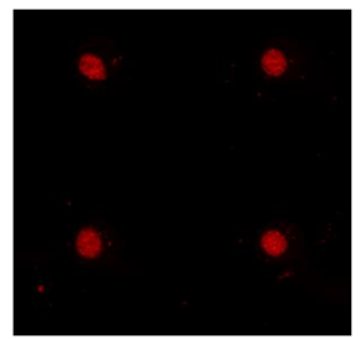

Day $1-$ E.coli treated sensors

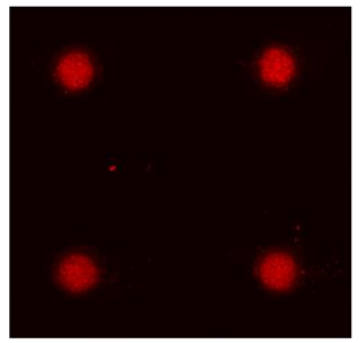

Day 7 - E.coli treated sensors

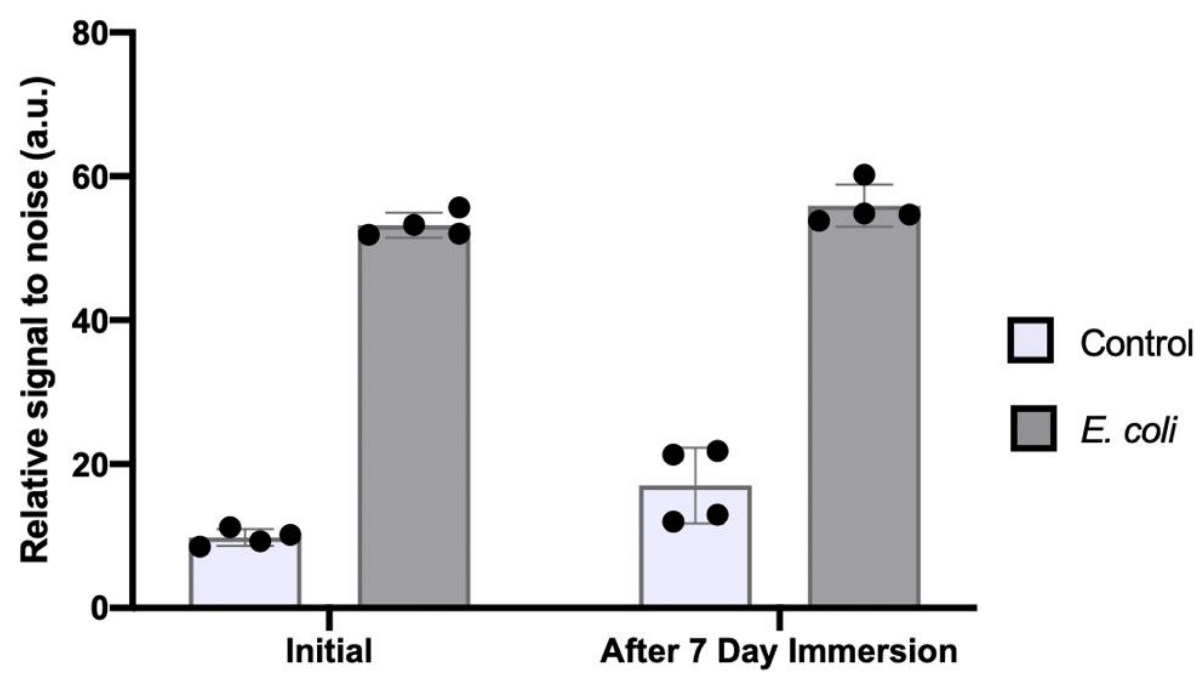

Figure S6. Long-term stability of the sensors. Microscope images associated with the data in Figure $4 \mathrm{~b}$ of the main manuscript. Images associated with Figure 4 in the manuscript. To ensure that the DNAzyme activity was retained after storage, the LISzyme surfaces were immersed in milk for seven days and then incubated with E. coli CEM derived from cultures with a concentration of $10^{5} \mathrm{CFU} / \mathrm{mL}$ These samples were then washed and imaged appropriately. 


\section{References}

(1) Aguirre, S.D.; Ali, M.M.; Salena, B.J.; Li, Y. A Sensitive DNA EnzymeBased Fluorescent Assay for Bacterial Detection. Biomolecules 2013, 3 (3), 563-577.

(2) Ali, M.M.; Aguirre, S.D.; Lazim, H.; Li, Y. Fluorogenic DNAzyme Probes as Bacterial Indicators. Angewandte Chemie 2011, 50 (16), 3835-3838. 